\title{
Coronavirus pandemic (COVID-19) - Clinical trials advice
}

\author{
Daniele Novara* \\ Italian pedagogue, Milan, Italy
}

There is currently an outbreak of respiratory disease caused by a novel coronavirus that was first detected in Wuhan City, Hubei Province, China, and that has now been detected in many locations internationally. COVID-19 pandemic may impact the conduct of clinical trials of medical products. Challenges may arise, for example, from quarantines, site closures, travel limitations, interruptions to the supply chain for the investigational product, or other considerations if site personnel or trial subjects become infected with COVID-19. These challenges may lead to difficulties in meeting protocol-specified procedures, including administering or using the investigational product or adhering to protocol-mandated visits and laboratory/ diagnostic testing.

Due to the Coronavirus situation most of European Regulatory Authority (Italy, Spain, UK, Finland, Denmark, etc.) and FDA provided some advise regarding the clinical trials during the pandemic. In this article I want to provide a summary of advises provided by the Regulatory Authority.

These measures are intended to preserve the activities of the trial as far as possible, guaranteeing the health care of the patients, protecting their safety and well-being and preserving the traceability of the actions implemented in this health emergency situation.

It is expected that the sponsor performs a thorough risk assessment of each individual ongoing trial and implements measures which priorities patient safety and data validity. In case these two conflicts, patient safety should take priority.

These risk assessments should be based on relevant parties' input and should be documented on an ongoing basis. The Sponsor should reassess risk as the situation develops. This reassessment should also be documented.

\section{Consideration}

\section{Patient safety}

Ensuring the safety of trial participants is paramount. Sponsors should consider each circumstance, focusing on the potential impact on the safety of trial participants, and modify study conduct accordingly.

The safety of a trial subject is at risk because they cannot complete key evaluations or adhere to critical mitigation steps, then consideration to discontinuing that subject must be discussed. The Sponsor, together with the investigator, based on a benefit / risk assessment that considers the characteristics of the trial and the circumstances of the participating sites, may interrupt the recruitment and even interrupt the treatment of the trial patients in order to avoid unnecessary risks and guarantee the best possible healthcare for patients. This analysis is especially pertinent in clinical trials involving immunosuppressant treatment and therefore an increased risk of infection, with no expectation of benefit for participants.

In the event of an interruption of the trial that leads to the cessation of treatment in part of the patients, the Sponsor would have to notify these measures as "urgent safety measures" explaining the measures taken to guarantee the alternative treatment of the patients by sending an Ad-Hoc report.

Such decisions will depend on specific circumstances, including the nature of the investigational product, the ability to conduct appropriate safety monitoring, the potential impact on the investigational product supply chain, and the nature of the disease under study in the trial.

\section{Protocol Deviation}

Any deviation from current practice must be proportionate, justifiable and clearly documented (see ICH GCP 5.0.4). The number of protocol deviations may increase during the transition period. It is important that these differences are clearly documented (see ICH GCP 4.5.3). Authorities will take a fair approach when reviewing derogation, as long as the interests of participants are taken into account and they are not exposed to undue risk.

\section{Patient visits/Site closure}

The sponsor (in cooperation with the Principal Investigator) should also consider whether physical visits can be converted to phone visits, postponed or cancelled completely to ensure that only strictly necessary visits are performed at sites. This consideration should also be part of the sponsor's risk assessment in relation to the COVID-19 pandemic.

In case it is not feasible for a site to continue participation at all, the sponsor should consider if the trial site should be terminated and how this can be done to best ensure patient safety and data validity.

If a site is closed to the public for COVID-19 containment measures, it should be carefully assessed if the clinical trial staff is able to guarantee the continuity of the trial itself. In case the site is unable to follow the patients undergoing the trial, the study should be temporarily halted or, where possible, enrolled patients should be transferred to the nearest active trial site. Information exchange between PIs must be assured, as well as the transmission of clinical documentation and

${ }^{*}$ Correspondence to: Daniele Novara, Italian pedagogue, Milan, Italy, Tel: 00393284480421; E-mail: danynovi@hotmail.it

Received: March 20, 2020; Accepted: April 03, 2020; Published: April 08, 2020 
other trial material (e.g. IMPs) between sites. Contacts between Sponsor and health structures involved must be updated according to new agreements. A site not authorized to participate in the specific clinical trial is not considered as suitable as back-up, since it is not active, it does not know the trial and could not ensure a proper therapeutic continuity for the patient.

\section{Site monitoring}

In order to reduce on-site activities, in particular on-site monitoring, appropriate alternative methods should be preferred. Alternative methods should be decided on the basis of a risk analysis, taking into account patient and data security, in agreement with the test sites, and the monitoring plan should be modified on the basis of accepted changes. Remote and central monitoring via the EDC system focusing on the most important data for subject safety and data quality may be an appropriate alternative.

Sharing of patient data and remote access of the Sponsor's representative to the electronic database of medical institutions is not acceptable due to the protection of particularly sensitive data and ethical considerations. It is important to emphasize that proper follow-up of these transitional measures once the situation has returned to normal is essential and includes, for example: an increase in the frequency and / or time of on-site monitoring to identify and manage any adverse effects of the transitional measures [1-8].

\section{Conclusion}

Sponsors, clinical investigators, and IECs should consider establishing and implementing policy and procedures, or revise existing policy and procedures, to describe approaches to be used to protect trial participants and manage study conduct during possible disruption of the study as a result of COVID-19 control measures at study sites. Changes to policy and procedures could address, but not be limited to, impact on the informed consent process, study visits and procedures, data collection, study monitoring, adverse event reporting, and changes in investigator(s), site staff, and/or monitor(s) secondary to travel restrictions, quarantine measures, or COVID-19 illness itself. Policy and procedures should be compliant with applicable (regional or national) policy for the management and control of COVID-19. Depending upon the nature of the changes described above, a protocol amendment may be required under the applicable regulations.
Sponsors should describe in appropriate sections of the clinical study report (or in a separate study specific document):

1. Contingency measures implemented to manage study conduct during disruption of the study as a result of COVID-19 control measures.

2. A listing of all participants affected by the COVID-19 related study disruption by unique subject number identifier and by investigational site, and a description of how the individual's participation was altered.

3. Analyses and corresponding discussions that address the impact of implemented contingency measures (e.g., trial participant discontinuation from investigational product and/or study, alternative procedures used to collect critical safety and/or efficacy data) on the safety and efficacy results reported for the study.

Robust efforts by sponsors, investigators, and IRBs/IECs to maintain the safety of trial participants and study data integrity are expected, and such efforts should be documented. As stated above, FDA recognizes that protocol modifications may be required, including unavoidable protocol deviations due to COVID-19 illness and/or COVID-19 control measures. Efforts to minimize impacts on trial integrity, and to document the reasons for protocol deviations, will be important.

\section{References}

1. MHRA Inspectorate (2020) Advice for Management of Clinical trials in relation to Coronavirus. Gail Francis and Kirsty Wydenbach (Posted).

2. Exceptional measures applicable to clinical trials to manage problems arising from the emergency by COVID-19 (2020) Spanish Agency for Medicines and Health Products (AEMPS).

3. Extraordinary measures for clinical trials due to COVID-19 (2020) Danish Medicine Agency.

4. Information on the continuity of clinical trials under COVID-19 (coronavirus) (2020) National Institute of Pharmacy and Nutrition of Hungary (OGYÉI)

5. COVID-19 Important announcements (2020) (ANMDMR) National Agency for Medicines and Medical Devices in Romania.

6. Emergency Measures For Clinical Trials Due To COVID-19 (2020) State Institute For Drug Control, Slovak Republic.

7. Clinical trials' management in Italy during the COVID-19 (coronavirus disease 19) (2020) AIFA Italian Medicines Agency.

8. FDA (2020) FDA Guidance on Conduct of Clinical Trials of Medical Products during COVID-19 Pandemic release on March 2020

Copyright: (C2020 Novara D. This is an open-access article distributed under the terms of the Creative Commons Attribution License, which permits unrestricted use, distribution, and reproduction in any medium, provided the original author and source are credited. 